\title{
AN EXERCISE TEST IN INTERMITTENT CLAUDICATION
}

\author{
BY \\ LAWSON MCDONALD AND ROBERT SEMPLE
}

From The Department of Medicine, The Middlesex Hospital

Received May 24, 1951

In most cases of intermittent claudication the diagnosis is apparent after the history and clinical examination have been completed. Although the history itself may be misleading, clinical examination is usually fruitful; the signs of obliterative arterial disease are present in most, and cases of anæmia and coarctation of the aorta that present with intermittent claudication should be easily diagnosed. However, in a number of cases the diagnosis remains doubtful. André-Thomas and Levy-Valensi (1918), and André-Thomas (1922) found in patients with intermittent claudication a diminution in oscillometric pulsations after exercise. Comroe (1923) finding a diminution in the peripheral pulses of intermittent claudication patients after exercise, believed that arterial spasm might be responsible even in the absence of obliterative arterial disease. Pearl (1937) reported six cases showing a similar phenomenon. Similar cases have been reported by Leary and Allen (1941) and by Lindqvist $(1945 a, 1945 b)$. The latter stressed the value of oscillometry after exercise in diagnosis; he believed that the diminution in pulsations which he found was evidence of vascular spasm, rather than a sequel of obliterative arterial disease, implying a functional rather than an organic lesion. Arterial spasm itself as a source of pain in intermittent claudication was denied by Lewis et al. (1931); Leriche (1937) and Ejrup (1948) further supported Lewis's view, as do our findings.

In 1944 Ejrup published an account of an instrument which after modification he considered would act as a tonoscillograph; he defined this as an instrument for measuring and recording variations in the pulsations and pressure in a vessel (Ejrup, 1948). His apparatus has the advantage of enabling continuous recordings to be made both before and after exercise. He studied the appearances of tonoscillographic tracings obtained before and after exercise in normal subjects and in those with intermittent claudication. Normal subjects showed an increase in the magnitude of pulsations and an apparent increase in blood pressure after exercise. Cases of intermittent claudication showed a decrease in the magnitude of pulsations after exercise, and an apparent fall in blood pressure distal to the site of arterial obstruction in the affected limbs. This phenomenon Ejrup termed the "inverse reaction." He also found the inverse reaction in some cases of anæmia (Ejrup, 1949), and of coarctation of the aorta; the inverse reaction was not found in cases of Raynaud's syndrome.

The following is an account of normal persons, patients with intermittent claudication, and those with pain in the leg due to other causes than obliterative arterial disease, studied with Ejrup's tonoscillograph before and after exercise. This investigation was made to evaluate tonoscillography after exercise in the diagnosis of intermittent claudication due to obliterative arterial disease, to correlate tonoscillographic and arteriographic findings, and to determine whether tonoscillography after exercise could be used in place of arteriography in the location of arterial obstruction. We further wished to discover whether the inverse reaction could be recognized clinically:

\section{ApPaRatus}

The apparatus is an automatic oscillograph. A cuff placed around the limb is automatically inflated and deflated with compressed oxygen, and pulsations in it during the inflation phase are 


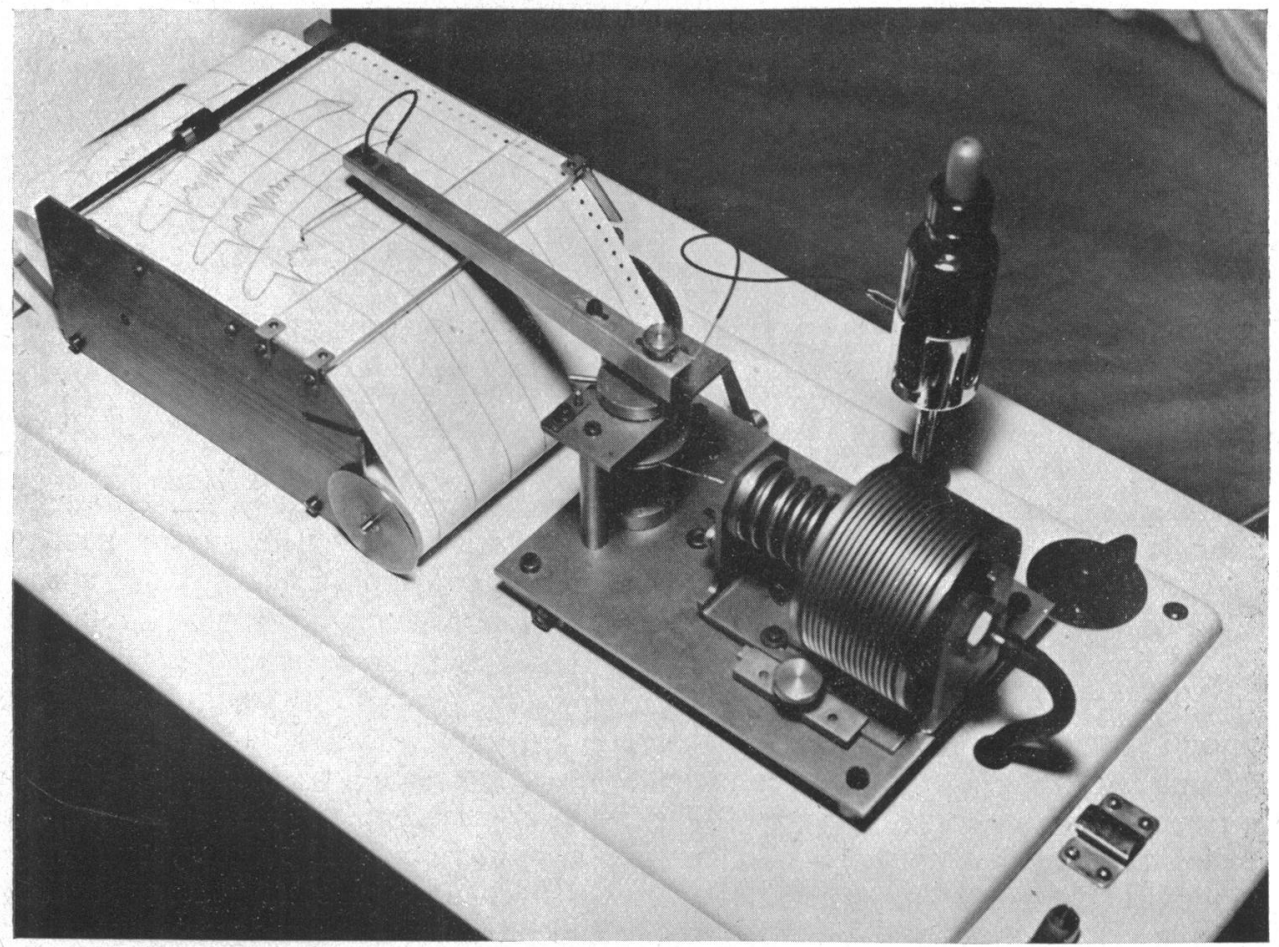

FIG. 1.-Ejrup's tonoscillograph. The writer is shown advancing vertically during the inflation phase, and recording pulsations in the horizontal direction.

transmitted, by means of a piezo-electric manometer and an amplifier, to a pen (Fig. 1). As the pressure increases the pen advances vertically and pulsations are recorded in the horizontal plane. The recording paper is marked with horizontal lines, and the apparatus calibrated so that these mark the pressure increase in the cuff in steps of $50 \mathrm{~mm}$. $\mathrm{Hg}$ from 0 to $300 \mathrm{~mm}$. A tracing is automatically obtained every 30 seconds.

\section{Methods}

Examinations are always made in the same room which varies only slightly in temperature $\left(18^{\circ}-22^{\circ} \mathrm{C}\right.$.). Before examination patients rest undisturbed on a rubber mattress for at least thirty minutes. The cuffs used (Fig. 2) are of three different sizes, varying in width and length. Each consists of an inflatable rubber ring surrounded by a firm cloth covering. On the outside of the latter is a row of numbered metal rungs, and on the end a hook. The cuff is tightly secured by engaging the hook on a rung.

The same rung is used in readings before and after exercise and the tightness of the cuff is thereby kept the same. The different sized rubber rings measure approximately $25 \times 8 \mathrm{~cm} ., 32 \times 10 \mathrm{~cm}$., and $61 \times 10 \mathrm{~cm}$. The smallest of the cuffs which will encircle the limb at the site being examined is used.

Recordings are made at three different levels of the limb; at the ankle, the calf, and the thigh. At the ankle the cuff is placed so that its lower border is level with the upper end of the medial malleolus. At the calf it is placed so that the middle of the cuff lies between the knee joint and the thickest part of the calf. At the thigh the middle part of the cuff is made to lie around the thinnest part of the thigh above the knee. Its lower edge then coincides with the upper margin of the patella. 


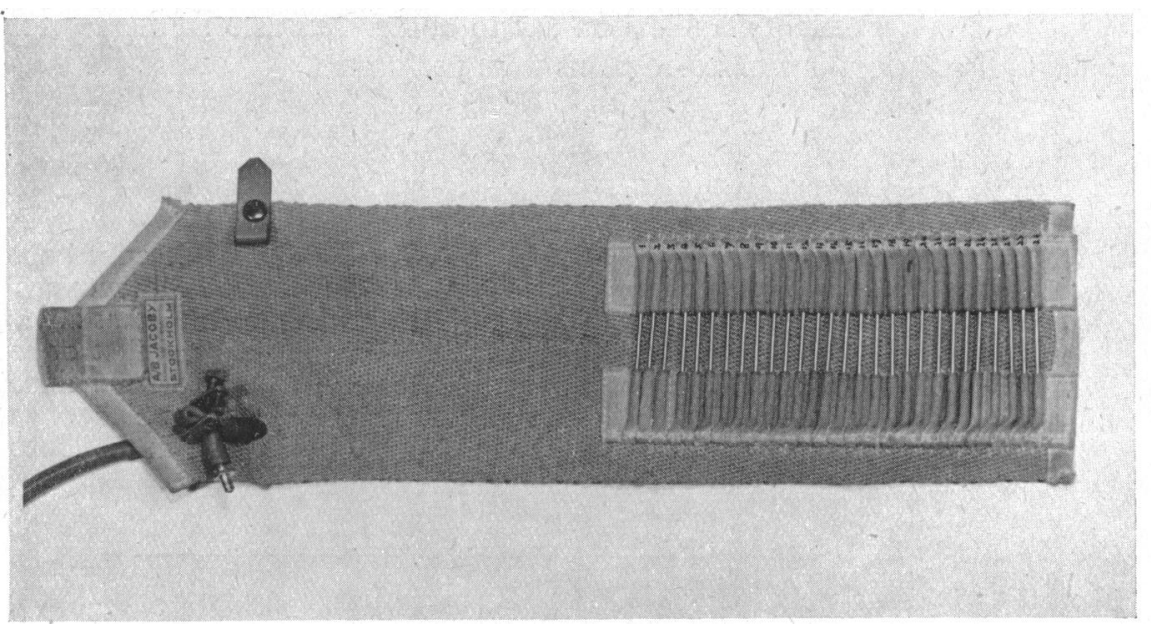

FIG. 2.-Cuff with numbered metal rungs. The inflatable rubber ring is on the inner side of the cloth covering.

In cases where the recording at the ankle is normal a tracing may be made at the foot; though owing to the shape of the foot and the difficulty in obtaining close apposition of the cuff to it, this is not always satisfactory. In cases of extensive arterial obstruction there may be insufficient pulsations to give satisfactory tracings, even with a high degree of amplification.

Following the practice of Ejrup (1948) recordings are first made at rest and the amplification adjusted so that the maximum horizontal deflection measures approximately $8 \mathrm{~mm}$. Experience has shown that this is the most satisfactory size of deflection. Two to four tracings are taken and the recording stopped. After tracings have been taken at rest the subject exercises. We have tried various methods of exercise for patients, including running and walking in a corridor, walking on a moving belt, using the limb to work an ergometer, and repeated dorsiplantar flexion of the foot with the leg in the horizontal position. The following are considered to be the most satisfactory types of exercise and have been adopted: for recordings from the thigh, calf, and foot the subjects if possible run slowly, or alternatively walk briskly, for up to $744 \mathrm{feet}$. This distance is usually covered in about 120 seconds. Sometimes the development of pain prevents their reaching this distance. In subjects who are unable to walk, dorsiplantar flexion of the foot at the rate of once a second for up to 90 seconds is performed, with the leg horizontal. This has been found to be sufficient exercise for ankle and foot tonoscillography. Control subjects never performed less exercise than patients with intermittent claudication.

After exercise the subject immediately lies down again, and a series of further tracings is commenced, care being taken to ensure that the lag period between the end of exercise and the start of the first tracing is never more than thirty seconds (see below). The subject rests undisturbed for thirty minutes between the end of a recording after exercise and the commencement of the next.

In attempting to elicit the inverse reaction clinically, subjects rest as above, and the dorsalis pedis and posterior tibial pulses are palpated. If these are present, dorsiplantar flexion of the foot is performed, as described for tonoscillography. Immediately after this exercise the dorsalis pedis and posterior tibial arteries are again palpated, and disappearance or diminution in their pulsations noted.

\section{SubJeCTS}

Three groups of subjects were studied:

(1) 42 normal subjects,

(2) 4 patients with pain in the leg not due to obliterative arterial disease, 
(3) 53 patients with intermittent claudication due to obliterative arterial disease; in 27 patients in this group femoral arteriograms or aortograms were performed.

\section{RESULTS}

Group 1. Normal subjects. Tonoscillography before and after exercise, as described above, was performed on forty-two subjects without symptoms of intermittent claudication, and without signs of peripheral vascular disease or any condition which might cause such pain. Ninety tonoscillograms after exercise were performed on these subjects. On comparing the magnitude of pulsations in the first or second tracing after exercise with those taken at rest the large majority showed an increase; the remainder showed no change. The level of pressure in the cuff at which the pulsations disappeared was higher or remained unchanged (Fig. 3). The relation of pulsations to possible blood pressure changes in the limbs will be considered more fully below.

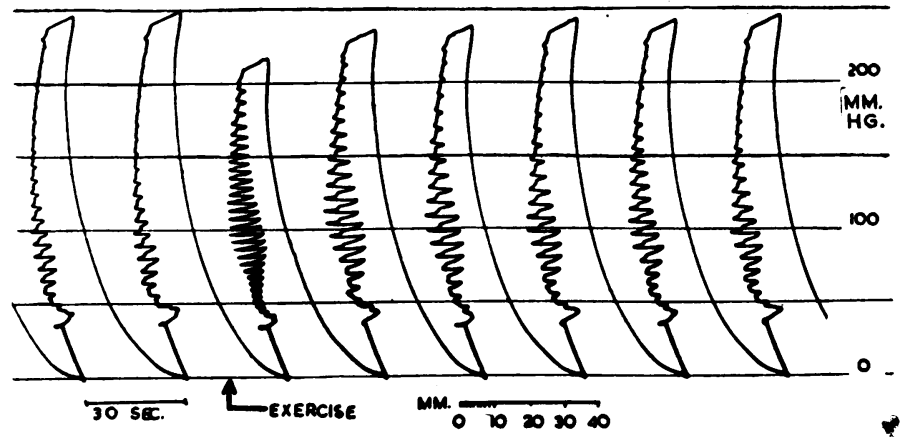

Fig. 3.-Tonoscillogram from right thigh of normal subject, showing increase in the magnitude of pulsations after exercise.

Some normal subjects show a decrease in the magnitude of pulsations in the first tracing after exercise. This phenomenon will be further considered later.

Group 2. Patients with pain in the leg not due to obliterative arterial disease. Four patients who were referred for investigation of pain in the leg, and in whom a diagnosis of intermittent claudication due to obliterative arterial disease was not made, form this group.

Case 1. M.B., man, aged 46 years. A poor witness, who gave a history of nine months' pain in the left leg, suggestive of intermittent claudication. There were no objective clinical or radiological signs of obliterative arterial disease, nor of any condition that might cause intermittent claudication. Varicose veins were present in the left lower leg. Femoral arteriography showed normal arteries throughout the left leg. Tonoscillography after exercise was completely normal. The pain in this man's leg was considered to be due partly to his varicose veins, and partly to psychogenic causes.

Case 2. H. L., man, aged 49 years. History of ten months' pain in the right leg which was considered to be suggestive of, but probably not, intermittent claudication. He had had poliomyelitis when aged three years. There were no objective clinical or radiological signs of obliterative arterial disease, nor of any condition that might cause intermittent claudication. There was wasting and shortening of the left leg from old poliomyelitis. Femoral arteriography showed normal arteries throughout the right leg. Tonoscillography after exercise was completely normal. The pain in this man's leg was considered to be connected with a grossly abnormal gait following poliomyelitis.

Case 3. H.H., man, aged 40 years. History of twelve months' pain and weakness in the legs, which was considered to be suggestive, but probably not, that of intermittent claudication. There were no objective clinical or radiological signs of obliterative arterial disease, nor of any condition which might cause intermittent claudication. Femoral arteriography showed normal arteries throughout the right leg. Tonoscillography after exercise was completely normal. This patient subsequently developed symptoms and signs of prolapse of the intervertebral disc between the sixth and seventh cervical vertebræ; it is considered possible that the pain in his leg may be related to the latter condition.

Case 4. M.B., woman, aged 35 years. A poor witness, who gave a history of two years' pain in the legs, suggestive of intermittent claudication. There were no objective clinical or radiological signs of obliterative arterial disease, nor of any condition that might cause intermittent claudication. Femoral arteriography 
showed normal arteries throughout the right leg. Tonoscillography after exercise was completely normal. No cause has been found for the pain in this patient's leg, but her unreliability as a witness has been increasingly apparent on repeated visits to hospital, also her tendency to symptoms of psychoneurotic illhealth.

In all these four patients clinical and radiographic evidence was against a diagnosis of intermittent claudication due to obliterative arterial disease, and the findings on tonoscillography after exercise were those of normal subjects

Group 3. Patients with intermittent claudication due to obliterative arterial disease. Tonoscillography before and after exercise was carried out in fifty-three patients in this group. In twenty-seven of these patients the diagnosis of obliterative arterial disease was confirmed by arteriography or aortography. On the fifty-three patients $\mathbf{2 8 8}$ tonoscillograms after exercises were recorded.

In all cases of intermittent claudication there was a decrease in the magnitude or a disappearance of pulsations compared with those obtained at rest (inverse reaction). This lasted at least during the first two tracings after exercise and was found at a definite level in the limb and persisted distal to that site (Fig. 4). Pulsations may not return to their amplitude before exercise for as long as thirty minutes, and disappear at a lower level of pressure in the cuff.

There was good correlation between the site of the obstruction demonstrated by arteriography and the level of the inverse reaction, which was present distal to gross narrowing or obstruction of a vessel. Thus of seven cases with gross narrowing or obstruction of the aorta or iliac arteries, six showed inverse reactions at the thigh level and below; while in the remaining case it was not possible

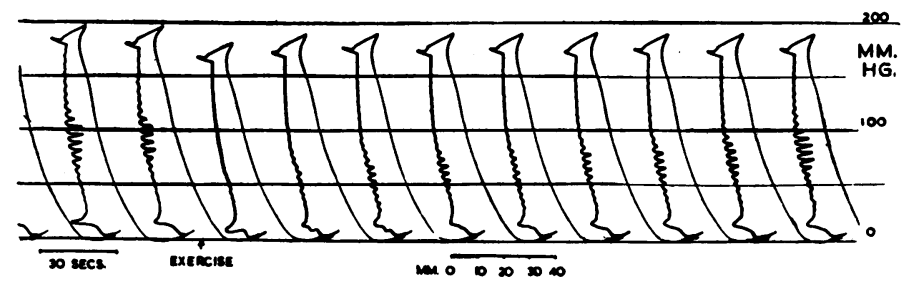

FIG. 4.-Tonoscillogram from right ankle of patient with intermittent claudication due to obliterative arterial disease, showing a decrease in the magnitude of pulsations after exercise.

to record any pulsations in the limb at all. Twenty cases on arteriography showed the limb vessels to be affected; in fourteen there was complete obstruction of a major vessel, and in six gross narrowing. The femoral artery was completely obstructed in ten of the fourteen (Cases 1,3 , $12,13,15,16,22,23,29,31$ ); all, except one (Case 29) of these showed inverse reactions at the level of the calf and distally. This exception showed normal reactions after exercise at the thigh and calf, and an inverse reaction at the ankle. Cases 3 and 12 showed in addition an inverse reaction at the thight. In Case 12 the inverse reaction at the thigh was of much shorter duration than in cases with aortic or iliac artery obstruction. Case 3 had a much less easily felt femoral pulse on the affected side, suggestive of an additional obstruction proximal to the femoral artery. Three (Cases 8, 44, 46) showed complete obstruction at the level of the popliteal artery, but not above; these showed inverse reactions at the ankle. Case 5 showed complete obstruction of one of the lower leg arteries, the anterior tibial, in the absence of more proximal complete obstruction; an inverse reaction was found only at the foot.

In six (Cases $6,28,34,35,37,39)$ there was narrowing of the limb arteries without complete obstruction; in one of these (Case 28) where the femoral artery only was affected, inverse reactions were found at the thigh level and distally. As in Case 12 the inverse reaction at the thigh was of much shorter duration than in cases with aortic or iliac artery obstruction. Case 35 showed narrowing of the femoral, popliteal and lower leg vessels: inverse reactions were present at the calf and ankle. In three (Cases 6, 34, 19) the femoral and lower leg vessels were affected; one (Case 39) showed inverse reactions at the ankle and foot (recordings were not made at the calf and thigh), 
another (Case 6) at the ankle, and the third (Case 34) at the foot only. In Case 37 only the posterior tibial artery was affected; an inverse reaction was present only at the foot.

Fig. 5 and 6 show the relation between tonoscillography after exercise and arteriography in all cases in which both examinations were made. A recording at the foot was usually only made in those cases in whom a normal reaction after exercise had been found at the ankle. In Cases 13 and 15 there were insufficient pulsations to record at the ankle and foot. In Case 39 recordings were not made at the thigh and calf. Cases in which aortography was performed are not included in Fig. 6.

Clinical Determination of the Inverse Reaction. In fifteen patients of Group 3 (intermittent claudication due to obliterative arterial disease), in whom the dorsalis pedis and posterior tibial arteries were palpable at rest, pulsations in these vessels were examined before and after exercise, as described above. All but two of these patients showed a diminution or absence of pulsations after exercise, comparable to the inverse reaction on tonoscillography after exercise, but often of shorter duration.

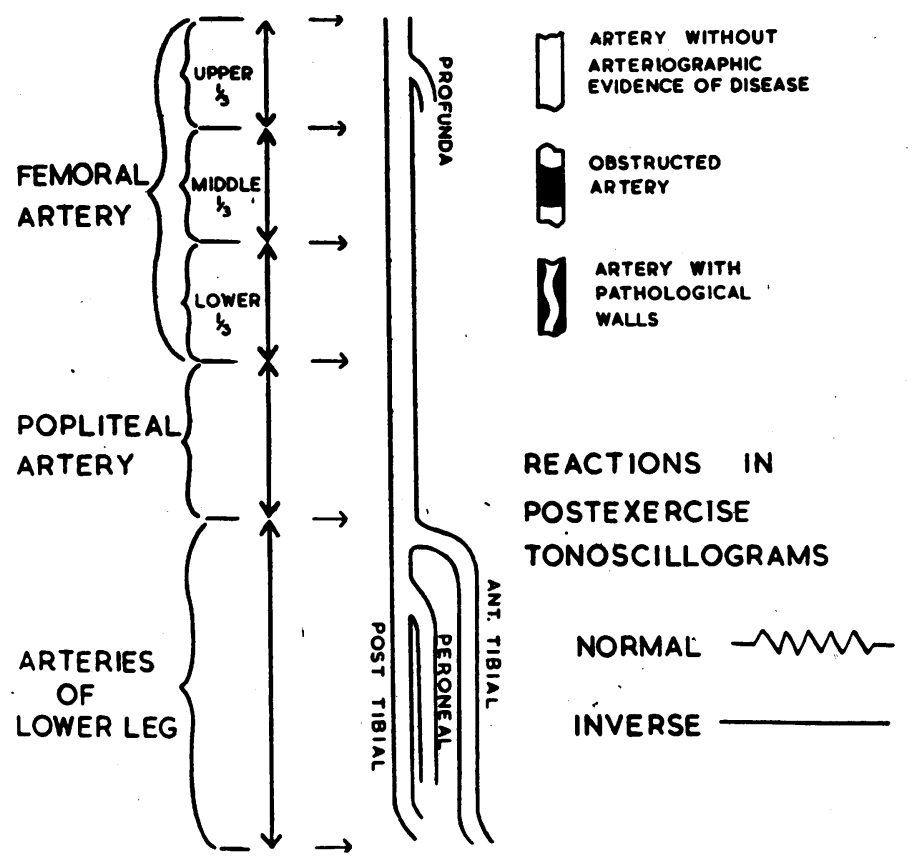

FIG. 5.-Key to diagrams comparing arteriographic and tonoscillographic findings.

\section{Discussion}

The Diagnosis of Intermittent Claudication due to Obliterative Arterial Disease. Ejrup (1948) described the inverse reaction as an invariable finding in patients with intermittent claudication, compared with the response found in normal subjects. Our results show its relation to intermittent claudication in cases due to obliterative arterial disease.

This condition may present considerable difficulty in diagnosis; the history obtained may be inconclusive, the peripheral pulses at rest may be easily felt and nutritional changes in the limbs absent. Boyd (1950) pointed out the difficulty which the diagnosis of intermittent claudication presents in some cases.

Tonoscillography after exercise may be used as a simple method of confirming a clinical diagnosis 

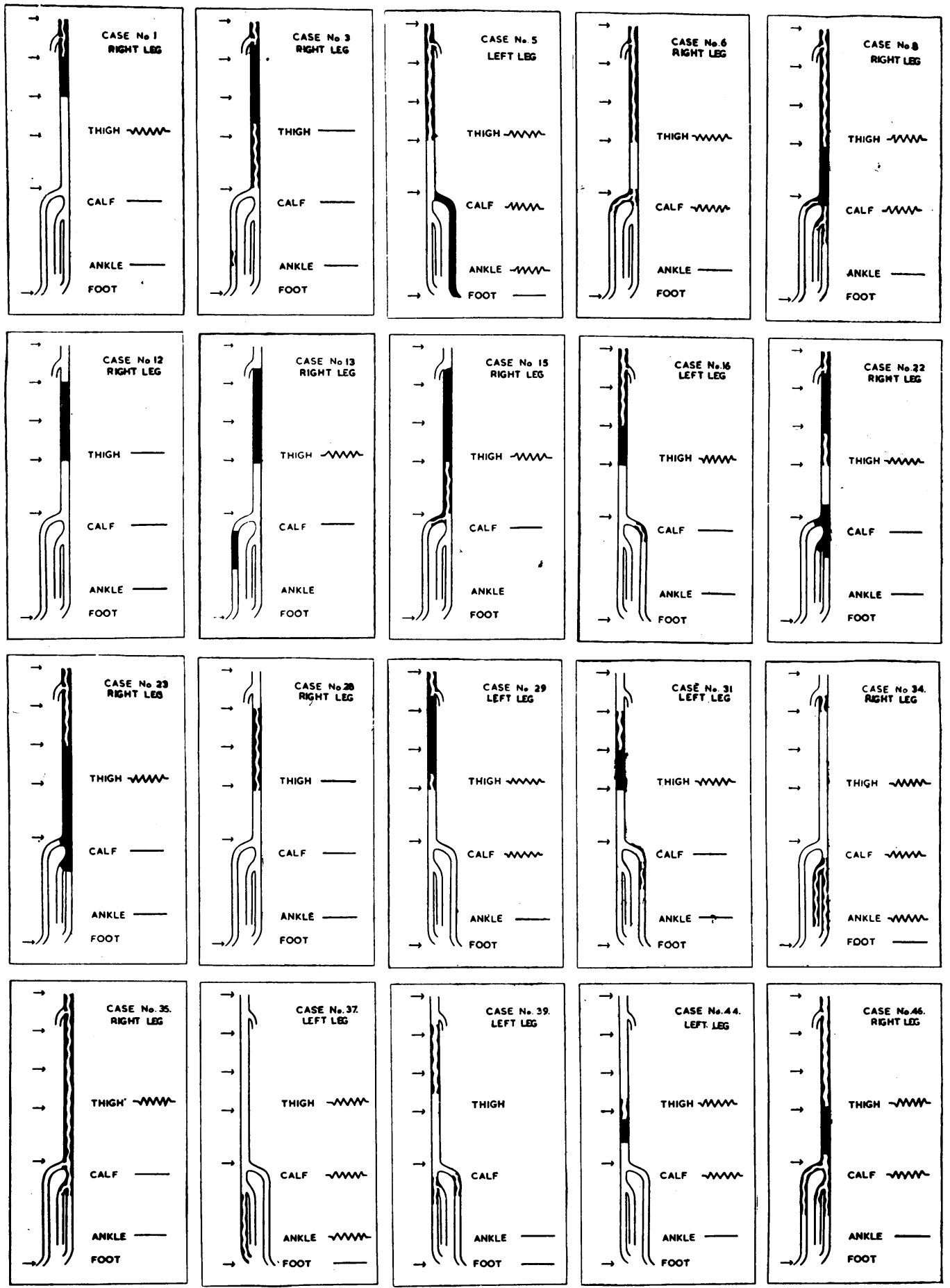

FIG. 6.-Comparison between arteriographic and tonoscillographic findings in 20 cases. 
of intermittent claudication due to obliterative arterial disease. In all cases the tonoscillographic findings should be considered in conjunction with the clinical picture, as other painful conditions of the leg such as osteoarthritis may be present in addition to arterial disease.

The results of clinical examination of the peripheral pulses before and after exercise above show that the tonoscillograph is not always necessary to demonstrate the inverse reaction, but we have not elicited it clinically in all cases where it was shown on tonoscillography. A negative finding clinically is not therefore as reliable an indication of the absence of obliterative arterial disease.

The Location of Arterial Obstruction. Tonoscillography after exercise will approximately indicate the site of the upper level of an arterial obstruction (see Fig. 6). Thus in cases with obstruction of the aortic or iliac arteries inverse reactions were found at the level of the thigh and distally; in cases with femoral artery obstruction, at the level of the calf and distally; in those with popliteal artery obstruction, at the ankle and foot; and in those with obstruction of a lower leg artery, only at the foot. There was one exception to the above in the present series; Case 29, with a completely obstructed femoral artery, had a normal reaction after exercise at the calf level. In cases of intermittent claudication due to arterial disease where the obstruction is incomplete, tonoscillography is a less reliable indication of the site of the disease; but all such cases investigated by us showed an inverse reaction at some level in the affected limb. It should be emphasized that a normal response after exercise has never been found distal to the level at which an inverse reaction has been recorded. As the inverse reaction will be found throughout the leg distal to a block, no information is obtained about the lower level of the obstruction. Should direct arterial operations such as those described by dos Santos (1947), Holden (1950), Lian and Welti (1950), and Reboul and Laubry (1950) be planned, arteriography or aortography will be necessary to define the limits of the arterial obstruction. If one leg is symptom free tonoscillography after exercise will indicate the presence or absence of arterial disease in it. With these limitations tonoscillography after exercise offers, therefore, an alternative to arteriography, and is particularly useful where the latter is contraindicated.

Mechanism of the Inverse Reaction. This will await investigation by other methods. Observations have shown that it is essential that the muscles between the site of arterial block and the recording cuff are exercised. Thus dorsiplantar flexion of the foot, sufficient to produce ischæmic pain in the calf and an inverse reaction at the ankle in cases with femoral artery obstruction, has failed to produce an inverse reaction at the calf, where this has subsequently been recorded after walking or by exercising the thigh muscles only.

The development of the pain of intermittent claudication is not necessary for the production of an inverse reaction. It has been shown that pulsations, as recorded by this method, diminish in patients with intermittent claudication after exercise. It is not considered that a fall in blood pressure after exercise can necessarily be inferred from these tonoscillographic tracings. Ejrup (1948) considered that the blood pressure could be approximately measured from the range of pulsations, with a standardized 8-mm. deflection, and that there was a fall in blood pressure, as well as a decrease in the magnitude of pulsations as part of the inverse reaction. In all our cases of intermittent claudication (Group 3) there was an apparent fall in blood pressure in the limb on the tonoscillographic tracings after exercise, as compared to those at rest before exercise. This may not represent a true fall in blood pressure, owing to the method of estimating blood pressure from the tracings. If pulsations become much diminished or absent it appears to us that it will be impossible to measure blood pressure, unless it is assumed that pulsations and blood pressure alter pari passu. No evidence has been found for this.

The difficulty in attempting to measure blood pressure and blood pressure changes by this method has been demonstrated by recording resting tonoscillograms in normal healthy subjects, and deliberately varying the magnitude of pulsations from tracing to tracing by altering the amplification (Fig. 7). When pulsations are absent or very small, the end points of the pulse pressure range and the systolic and diastolic blood pressures are difficult if not impossible to determine.

Ejrup's method might be more accurately described as oscillography after exercise than as 
tonoscillography after exercise. The prefix ton- was added to denote the supposed function of measuring blood pressure. It has been retained by the present authors to identify the method.

The inverse reaction found in patients with intermittent claudication may be an accentuation of a normal response to exercise. We have found that after exercising normal healthy young adults to points of extreme leg exhaustion, by making them run " on the spot" at full speed for as long as they can, an "inverse reaction" at the ankle may be demonstrated. This very severe degree of exercise was far in excess of exercise necessary to produce an inverse reaction in the claudication group. Fig. 8 shows an inverse reaction produced in a healthy young subject after very severe exercise.

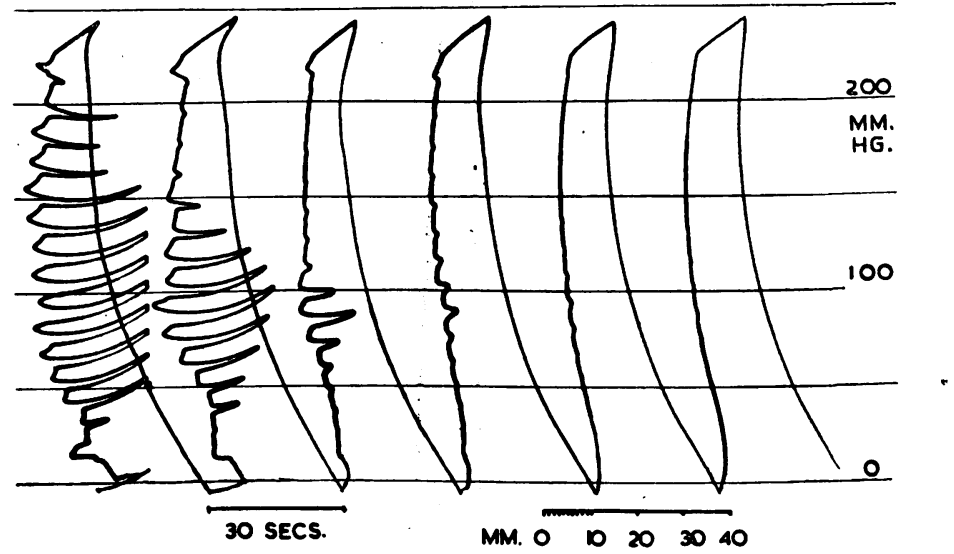

Fig. 7.-Tonoscillogram from right ankle of normal subject. The magnitude of pulsations has been decreased progressively in each tracing from left to right, by altering the amplification control on the apparatus. The difficulty in attempting to measure blood pressure from tonoscillograms is apparent from these tracings (see text). The subject's blood pressure in the right leg was, by the auscultatory method, 145/95.

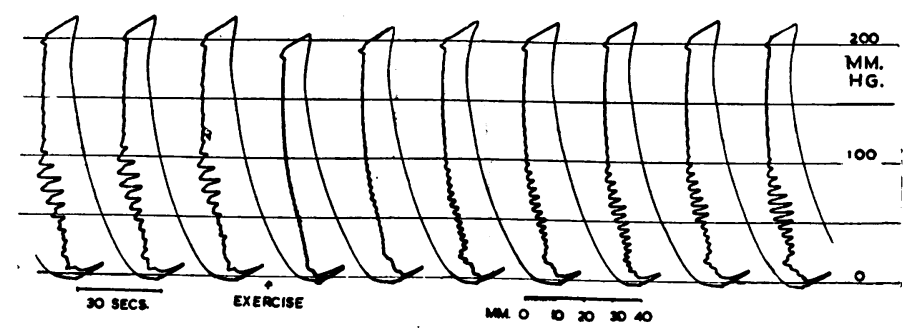

FIG. 8.-Tonoscillogram from right ankle of normal subject, showing " inverse reaction" produced after very severe exercise (see text).

A normal reaction was produced in the same subject after the most strenuous grade of exercise given to the intermittent claudication group, and it should be emphasized that in no control subject was an inverse reaction produced by the maximum degree of exercise required to produce it in any patient with intermittent claudication. The decrease in the magnitude of pulsations which some normal subjects show in the first tracing after much less exercise, further supports the possibility that the inverse reaction found in patients with intermittent claudication may be an accentuation of a normal response to exercise. All our arteriographed cases in Group 3 showed arterial obstruction and we have found no record of a proven case of intermittent claudication due to arterial spasm in the absence of obliterative arterial disease. Further work is being done with the object of determining the mechanism of the inverse reaction. 


\section{SUMMARY}

An exercise test, which may be performed either clinically or using Ejrup's tonoscillograph, is described and its use in the diagnosis of intermittent claudication is discussed. Forty-two normal subjects, four patients with pain in the legs not due to obliterative arterial disease, and fifty-three patients with intermittent claudication due to obliterative arterial disease have been studied by this method. In twenty-seven of the last group the diagnosis was confirmed by either arteriography or aortography. Pulsations in limb vessels after moderate exercise are found to increase or remain the same in normal subjects, and to decrease in those with intermittent claudication (the inverse reaction). The value of the finding of the inverse reaction in patients with intermittent claudication is discussed.

It is a pleasure to acknowledge the help and encouragement that we have received from Professor A. Kekwick in this work, and for his advice and that of Dr. John Goodwin in the preparation of the paper. We wish to thank members of the Consultant Staff of the Middlesex Hospital and others who have referred cases to us, Dr. David Sutton of the X-ray Diagnosis Department who performed the femoral arteriograms, also the secretaries, hospital photographer and hospital artist for their valuable assistance, and all those who acted as control subjects. We are indebted to the Board of Governors of the Middlesex Hospital who defrayed part of the cost of this investigation, and finally to. Dr. Borje Ejrup of Stockholm for his very kind co-operation at all times.

\section{REFERENCES}

André-Thomas, (1922). Pr. méd., 2, 1049.

and Levy-Valensi, J. (1918). Paris Méd., 27, 58.

Boyd, A. M. (1951). Proc. Roy. Soc. Med., 44 (in the press).

Comroe, J. H. (1923). Ann. clin. Med., 1, 313.

Dos Santos, R. (1947). Bull. Schweiz. Akad. med. Wiss., 3, 1.

Ejrup, B. (1944). Nord. Med., 23, 1770.

- (1948). Acta med. scand., 130, Suppl. 211.

(1949). Personal communication.

Holden, W. D. (1950). Surgery, 27, 417.

Leary, W. V., and Allen, E. V. (1941). Amer. Heart J., $22,719$.

Leriche, R. (1937). La Chirurgie de la Douleur, Paris, p. 257.

Lewis, T., Pickering, G. W., and Rothschild, P. (1931). Heart, 15, 359.

Lian, C., and Welti, H. (1950). Sem Hôp., Paris, 26, 3282.

Lindqvist, T. (1945a). Acta med. scand., 121, 32.

(1945b). Acta med. scand., 121, 409.

Pearl, F. L. (1937). Amer. J. med. Sci., 194, 505.

Reboul, H., and Laubry, P. (1950). Proc. Roy. Soc. Med., 43, 547. 